\title{
APPLICATION OF A METHOD OF SZEMEREDI
}

\author{
by H. HALBERSTAM
}

To Robert Rankin on his 70th Birthday

1. Let $\mathscr{B}=\left\{b_{i}: b_{1}<b_{2}<\ldots\right\}$ be an infinite sequence of positive integers that exceed 1 and are pairwise coprime, so that

$$
\left(b_{i}, b_{j}\right)=1, \quad i \neq j
$$

Assume also that

$$
\sum_{i=1}^{\infty} \frac{1}{b_{i}}<\infty .
$$

Let $\mathscr{A}=\mathscr{A}_{\mathscr{B}}$ denote the sequence of $\mathscr{B}$-free numbers, that is, of positive integers divisible by no element of $\mathscr{B}$. This concept, generalizing square-free and $k$-free numbers, derives from Erdös [2] who proved in 1966 that there exists a constant $c, 0<c<1$, independent of $\mathscr{B}$, such that the interval $\left(x, x+x^{c}\right)$ contains elements of $\mathscr{A}$ provided only that $x$ is large enough. This result of Erdös was shown by Szemeredi [7] in 1973 to hold with $c=\frac{1}{2}+\varepsilon$, if $x \geqslant x_{0}(\varepsilon, \mathscr{B})$, and quite recently Bantle and Grupp [1] have sharpened Szemeredi's result to $c=9 / 20+\varepsilon$.

The purpose of this note is to show how the method of Szemeredi can be used to derive, virtually without change, the following result.

THEOREM. Let $k$ be a positive integer and let $h$ satisfy $1 \leqslant h<k,(h, k)=1$. Given $\delta>0$, there exists a $\mathscr{B}$-free number a such that

$$
a \equiv h \bmod k, \quad a \leqslant k^{2+\delta}
$$

provided only that $k \geqslant k_{0}(\varepsilon, \mathscr{B})$.

Define, as (1.2) permits us to do,

$$
\beta=\prod_{i=1}^{\infty}\left(1-\frac{1}{b_{i}}\right)
$$

and denote by $s$ the least positive integer so that

$$
\sum_{i=s+1}^{\infty} \frac{1}{b_{i}}<\frac{1}{100} \varepsilon \beta .
$$

It is easy to see that, without any loss of generality, one may assume $b_{1}, \ldots, b_{s}$ to be prime. We shall use the letters $p$ and $q$, with or without suffices, exclusively to denote primes.

We shall prove the theorem by showing that, actually, there exist at least $(1 / 20) \varepsilon \beta k^{1+\varepsilon} \mathscr{B}$-free numbers $a \leqslant k^{2+\varepsilon}$ in the arithmetic progression $h \bmod k$, provided that $k$ is large enough.

Glasgow Math. J. 27 (1985) 81-85. 
2. Proof of the theorem. The natural approach would be to develop an argument to show that the set $\left\{n: 1 \leqslant n \leqslant k^{2+\varepsilon}, n \equiv h \bmod k\right\}$ contains elements of $\mathscr{A}$ if $k$ is large enough. Instead, following Szemeredi, we narrow attention at the outset to a subset of these integers each having a large prime factor. It turns out that this surrender of advantage is more than compensated by the increased difficulty of having such integers divisible by large elements of $\mathscr{B}$.

Accordingly, let

$$
\mathscr{P}=\left\{p: 2 k^{1+\varepsilon / 2}<p<k^{1+\varepsilon}, p \notin \mathscr{B}, p \nmid k\right\},
$$

and focus on the set of integers

$$
\mathscr{C}=\left\{n: 1 \leqslant n \leqslant k^{2+\varepsilon}, n \equiv h \bmod k, n \text { divisible by a prime of } \mathscr{P}\right\} .
$$

If an integer $n$ in $\mathscr{C}$ were to have two prime divisors from $\mathscr{P}$ we should have $k^{2+\varepsilon} \geqslant n>$ $\left(2 k^{1+\varepsilon / 2}\right)^{2}$, a contradiction. Hence $\mathscr{P}$ induces the partition

where

$$
\mathscr{b}=\bigcup_{p \in \mathscr{P}} \mathscr{C}^{(p)}
$$

$$
\mathscr{C}^{(p)}:=\left\{n: 1 \leqslant n \leqslant k^{2+\varepsilon}, n \equiv h \bmod k, n \equiv 0 \bmod p\right\} .
$$

Moreover, if $\mathscr{C}_{1}$ now denotes the number of elements of $\mathscr{C}$ divisible by none of $b_{1}, \ldots, b_{s}$, then the cardinality $\left|\mathscr{C}_{1}\right|$ of $\mathscr{C}_{1}$ is given by

$$
\left|\mathscr{C}_{1}\right|=\sum_{p \in \mathscr{P}}\left|\mathscr{C}_{1}^{(p)}\right|=\sum_{p \in \mathscr{P}}\left|\left\{m: 1 \leqslant m \leqslant k^{2+\varepsilon} p^{-1}, m \equiv h p^{\prime} \bmod k,\left(m, b_{1} \ldots b_{s}\right)=1\right\}\right|
$$

where the interpretation of $\mathscr{C}_{1}^{(\mathrm{p})}$ is obvious and $p^{\prime}=p^{\prime}(k)$ is the inverse of $p$ modulo $k$, i.e. $p p^{\prime} \equiv 1 \bmod k$.

LEMMA 1. If $k$ is sufficiently large,

$$
\left|\mathscr{C}_{1}\right| \geqslant \frac{\varepsilon}{10} \beta k^{1+\varepsilon}
$$

Proof. By (2.4) and the definition of $\mathscr{P}$, which guarantees that $\left(p, b_{1} \ldots b_{s}\right)=1$ when $p \in \mathscr{P}$, we have

$$
\begin{aligned}
\left|\mathscr{C}_{1}^{(p)}\right| & =\sum_{\substack{d \mid b_{1} \ldots \ldots b_{s} \\
(d, k)=1}} \mu(d) \sum_{\substack{1 \leq m \leq k^{2+e} / p \\
m=h p^{\prime m o d} k \\
m=0 \text { modd }}} 1 \\
& =\sum_{\substack{d \mid p_{1} \ldots \ldots p_{s} \\
(d, k)=1}} \mu(d)\left(\frac{k^{1+\varepsilon}}{p d}+\theta_{p, d}\right), \quad\left|\theta_{p, d}\right|<1, \\
& \geqslant \frac{k^{1+\varepsilon}}{p} \sum_{\substack{d \mid b_{1} \ldots b_{s} \\
(d, k)=1}} \frac{\mu(d)}{d}-2^{s} \\
& =\frac{k^{1+\varepsilon}}{p} \prod_{\substack{i=1 \\
b_{i} \chi k}}^{s}\left(1-\frac{1}{b_{i}}\right)-2^{s} \\
& \geqslant \frac{k^{1+\varepsilon}}{p} \beta-2^{s} .
\end{aligned}
$$


Hence, by (2.4),

$$
\begin{aligned}
\left|\mathscr{C}_{1}\right| & \geqslant \beta k^{1+\varepsilon} \sum_{p \in \mathscr{P}} \frac{1}{p}-2^{s} \pi\left(k^{1+\varepsilon}\right) \\
& \geqslant k^{1+\varepsilon}\left\{\beta \sum_{p \in \mathscr{P}} \frac{1}{p}-\frac{2^{s+1}}{\log k}\right\} .
\end{aligned}
$$

But

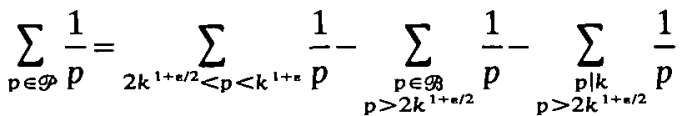

$$
\begin{aligned}
& \geqslant \frac{\varepsilon}{5}+O\left(\frac{1}{\log k}\right)-\sum_{i>s} \frac{1}{b_{i}}-k^{-1-\varepsilon / 2} \omega(k)
\end{aligned}
$$

if $k$ is large enough (to ensure that $k^{1+\varepsilon / 2} \geqslant b_{s+1}$ ), where $\omega(k)$ is the number of distinct prime factors of $k$; so that, by (1.4),

$$
\sum_{p \in \mathscr{P}} \frac{1}{p} \geqslant \frac{\varepsilon}{5}-\frac{\varepsilon \beta}{100}+O\left(\frac{1}{\log k}\right)
$$

and

$$
\left|\mathscr{C}_{1}\right| \geqslant \beta k^{1+\varepsilon}\left\{\frac{\varepsilon}{5}-\frac{\varepsilon}{100}+O\left(\frac{1}{\log k}\right)\right\} \geqslant \frac{\beta \varepsilon}{10} k^{1+\varepsilon}
$$

for all sufficiently large $k$, as required.

It follows from Lemma 1 that if $\mathscr{C}_{0}$ denotes the set of elements of $\mathscr{C}$ having no divisors from $\mathscr{B}$, then

$$
\left|\mathscr{C}_{0}\right| \geqslant\left|\mathscr{C}_{1}\right|-\left|\mathscr{C}_{2}\right|-\left|\mathscr{C}_{3}\right| \geqslant \frac{\varepsilon \beta}{10} k^{1+\varepsilon}-\left|\mathscr{C}_{2}\right|-\left|\mathscr{C}_{3}\right|
$$

where $\mathscr{C}_{2}$ is the set of all those integers in $\mathscr{C}$ that are divisible by an element $b$ in $\mathscr{B}$ of 'intermediate' size, i.e. one satisfying

$$
b_{s+1} \leqslant b \leqslant k^{1+\varepsilon} \text {, }
$$

and $\mathscr{C}_{3}$ is the set of all integers belonging to $\mathscr{C}_{1}$ that have a large factor $b$ from $\mathscr{B}$, i.e. satisfying $b>k^{1+\varepsilon}$. We shall prove that $\left|\mathscr{C}_{2}\right|+\left|\mathscr{C}_{3}\right|$ is relatively small.

LEMMA 2. We have $\left|\mathscr{C}_{2}\right| \leqslant \frac{\varepsilon \beta}{50} k^{1+\varepsilon}$. 
Proof. We argue quite crudely. We have that

$$
\begin{aligned}
\left|\mathscr{C}_{2}\right| & \leqslant \sum_{\substack{b_{s+1} \leq b \leq k^{1+e} \\
(b, k)=1}} \sum_{\substack{1 \leqslant n \in k^{2+e} \\
n=h \bmod \\
n=0 \bmod b}} 1 \\
& \leqslant \sum_{\substack{b_{s+1} \leq b \leq k \leq 1+\varepsilon \\
(b, k)=1}}\left(\frac{k^{1+\varepsilon}}{b}+1\right) \\
& \leqslant 2 k^{1+\varepsilon} \sum_{b \geqslant b_{s+1}} \frac{1}{b}<\frac{\varepsilon \beta}{50} k^{1+\varepsilon}
\end{aligned}
$$

by (1.4), and this completes the proof of the lemma.

Lemma 3. We have $\left|\mathscr{C}_{3}\right| \leqslant \frac{1}{2} k^{1+\varepsilon / 2}$.

Proof. Suppose $n$ is counted in $\mathscr{C}_{3}$. Then $n$ is divisible by a (unique) prime $p$ from $\mathscr{P}$, and $n$ is divisible also by an element $b>k^{1+\varepsilon}$ from $\mathscr{B}$. This cannot happen if $(b, p)=1$, for then $n \geqslant b p>k^{1+\varepsilon} \cdot 2 k^{1+\varepsilon / 2}=2 k^{2+3 \varepsilon / 2}$, a contradiction. Hence the $b$ dividing $n$ is composite and divisible by a $p>2 k^{1+\varepsilon / 2}$. Writing $b=l p$, we have $1<l<\frac{1}{2} k^{1+e / 2}$; and given such an integer $l$, there is, by (1.1), at most one $b \in \mathscr{B}$ divisible by $l$. Hence there are at most $\frac{1}{2} k^{1+\varepsilon / 2}$ available choices of $b$. Finally, given such a $b$, if $1 \leqslant n \leqslant k^{2+\varepsilon}, n \equiv h \bmod k$ and $n \equiv 0 \bmod b$ with $b>k^{1+\varepsilon}$, there is at most one such $n$. This proves the lemma.

We are now able to complete the proof of the theorem. By (2.5) and Lemmas 2 and 3 we have

$$
\left|\mathscr{C}_{0}\right| \geqslant \frac{\varepsilon}{10} \beta k^{1+\varepsilon}-\frac{\varepsilon}{50} \beta k^{1+\varepsilon}-\frac{1}{2} k^{1+\varepsilon / 2} \geqslant \frac{\varepsilon}{20} \beta k^{1+\varepsilon}
$$

if $k$ is large enough. Thus the theorem is proved, in a quantitative form.

3. Some concluding remarks. If we replace condition (1.2) by the more demanding

$$
B(x):=\left\{\{b \in \mathscr{B}: b \leqslant x\} \mid \ll x^{\theta},\right.
$$

where $0<\theta<1$, we can, with only a little more trouble, replace the exponent $2+\varepsilon$ in the theorem by $1+\theta+\varepsilon$. Thus when $\mathscr{B}$ is the sequence of squares of primes and $\mathscr{A}_{\mathscr{B}}$ is the sequence of squarefree numbers, we obtain the exponent $(3 / 2)+\varepsilon$ which is very close to the best that was known until the recent work of Heath-Brown [3]. While it is unlikely that one can emulate Heath-Brown's delicate argument in the more general situation, I do believe that the theorem itself can be improved a little, in the spirit of [1].

The condition (1.1) can be relaxed somewhat in the theorem. For such and other variations of Szemeredi's theorem see Narlikar and Ramachandra [4].

\section{REFERENCES}

1. G. Bantle and F. Grupp, On a problem of Erdös and Szemeredi, J. Number Theory, to appear. 
2. P. Erdös, On the difference of consecutive terms of sequences defined by divisibility properties, Acta Arith. 12 (1966), 175-182.

3. D. R. Heath-Brown, The least square-free number in an arithmetic progression, J. Reine Angew. Math. 332 (1982), 204-220.

4. H. J. Narlikar and K. Ramachandra, Contributions to the Erdös-Szemeredi theory of sieved integers, Acta Arith. 38 (1980), 157-165.

5. E. Szemeredi, On the difference of consecutive terms of sequences defined by divisibility properties II, Acta Arith. 23 (1973), 359-361.

UNIVERSTTY OF IllinOIS at URBANa-ChampaIGN

Department of Mathematics

1409 West Green Street

URBANA

ILLINOIS 61801

U.S.A. 